\title{
Targeted Inspection of Environmental Mycological Load for Mitigation of Indoor Mold toward Improved Public Health
}

Segula Masaphy ${ }^{1,2 *}$ and Rinat Ezra ${ }^{3}$

${ }^{1}$ Migal-Galilee Research Institute, Kiryat Shmona, Israel

${ }^{2} \mathrm{Tel}$ Hai College, Kiryat Shmona, Israel

${ }^{3}$ Queen Mary University London, UK

\begin{abstract}
Fungi and their toxic metabolites have been pinpointed as possible causes of sick building syndrome, an illness associated with poor indoor ventilation. Certain risk groups, such as hospitalized patients, the elderly and young children, tend to be more susceptible. Substantial effort has been devoted to establishing optimal fungal monitoring techniques and identification protocols. This overview screens the available monitoring and identification methods which may provide qualitative or quantitative information about the presence of particular fungi or their secondary metabolites/ mycotoxins and evaluation of their effect on the potential improvement in the associated health symptomology and the establishment of the pathophysiological mechanism involved in this process. Certain target locations, where mycological contamination is more likely to occur and certain risk groups that are in need of stricter environmental inspection and more refined fungal monitoring and identification protocols, are addressed. In assessing the impact of environmental inspection, there is no gold standard for the expected response rate in terms of fungal load reduction and its significance. This lack of standards and the limitations in associating specific fungal contamination with health effects may be related to the determination of indoor fungal load. This could be the result of either reporting biases of dampness or the choice of method used to monitor fungal load. Refined fungal monitoring and identification protocols are suggested for the more specific targeting of fungal isolates, their identification and quantification.
\end{abstract}

Keywords: Environmental inspection; Indoor mycological load; Mycotoxin; Pathogenicity; Sick building syndrome

\section{Highlights}

- Fungi and toxic metabolites indoors are associated with diverse health risks.

- Identification and quantitative monitoring of fungi and mycotoxins are reviewed.

- Cost effectiveness of these techniques relative to health risks is discussed.

- Monitoring of fungal load and associated health burden is considered.

- Objective measures to validate the impact of mitigating the fungal load on the health symptomology are considered.

\section{Introduction}

There is a growing body of evidence in Europe implicating a correlation between dampness and mold in buildings and increased risk of adverse health effects to building occupants $[1,2]$. There are similar reports in the United States (US) [3)] Mudarri and Fisk [4] estimated that of the 21.8 million people reported to have asthma in the US, approximately 4.6 (2.7-6.3) million cases can be attributed to dampness and mold exposure. The total annual asthma cost attributable to exposure to dampness and mold, calculated by multiplying the selected estimate of costs by the attributable fraction of $21 \%$, is approximately $\$ 3.5$ billion [4]. Moreover, reported cases of hospital-acquired fungal infections in hospitalized and immunosuppressive patients are on the rise [5-8]. Other groups at risk, such as the elderly [9] and young children [10,11], have been noted for their susceptibility to the harmful effects of indoor air and fungi. Both direct fungal infection (mycosis) [7] and toxigenic effects of fungal mycotoxins have been reported [12-16]

This rising epidemiological phenomenon requires preventive activities, and mitigation of the contaminated sources. These include the ability to define and quantify the mycological load and harmful sources, i.e., the types of fungi and their toxins, to determine their reduction upon treatment and to translate the fungal risk base into an environmental health policy that will include guidelines for monitoring fungal load and the associated health burden. However, regulations and standards regarding mycological load for prevention or mitigation strategies are limited $[17,18]$. In general, there is no gold standard for clinically significant fungal loads or fungal load reduction based on a comparison between treated and untreated moldy and damp environments. Environmental inspections (EIs) of mycological load have been suggested using a range of fungal-detection techniques, e.g. open Petri dish (OPD) versus active air sampling $[19,20]$ and qualitative identification and quantitative monitoring of diverse fungal species and their mycotoxins [21-24].

In an attempt to address this lack of standards, this overview summarizes the overall health risks in damp and moldy indoor environments, as well as the pathogenicity and mycotoxigenicity of specific fungi and mycotoxins in higher-risk environments. The diverse methodologies involved in identifying and quantifying the specific or overall mold and fungal load or specific fungal species and mycotoxins and their limitations, are also discussed. The efficacy of EIs and sophisticated air sampling and analysis techniques, such as fungal DNA analyses, and the use of more traditional approaches such

*Corresponding author: Segula Masaphy, Migal-Galilee Research Institute Kiryat Shmona, Israel, Tel: 972-4-6953519/594; Fax: 972-4-694498; E-mail: segula@migal.org.il

Received September 24, 2016; Accepted October 10, 2016; Published October 19,2016

Citation: Masaphy S, Ezra R (2016) Targeted Inspection of Environmenta Mycological Load for Mitigation of Indoor Mold toward Improved Public Health. J Microb Biochem Technol 8: 449-458. doi: 10.4172/1948-5948.1000324

Copyright: @ 2016 Masaphy S, et al. This is an open-access article distributed under the terms of the Creative Commons Attribution License, which permits unrestricted use, distribution, and reproduction in any medium, provided the original author and source are credited. 
the OPD method and microscopic analysis, are reviewed in relation to monitoring of the indoor mycological load. The integration of better validated screening criteria and occupants' subjective evaluations is indicated as a means of assessing the impact of, monitoring and mitigating mycological load.

\section{Overall Risks in Damp and Moldy Indoor Environments}

A growing body of evidence reported from Europe and the US shows correlations between dampness and mold in buildings and the risk of adverse health effects to the buildings' occupants, especially the respiratory system, although a much broader array of health outcomes has been reported [13]. The skin or gastrointestinal tract may become infested with mold and fungal substrates, and inhalation of fungal units and spore-borne toxins are important sources of exposure [7]. Nevertheless, health problems caused by inhaled mycotoxins tend to be more serious than those caused by mycotoxins absorbed via the digestive tract or skin [13-16]. Creasia et al. [25] showed that mycotoxins can be 10 times more toxic through inhalation than by systemic administration in mice. However, only fungal metabolites that can become airborne are relevant to human inhalational exposure. In a study by Smith et al. [26], 47\% of the fungal isolates from the air flora of a damp building displayed spore-associated cytotoxic properties. This emphasizes the potential health hazards of continuous inhalation of toxigenic fungal propagules and their toxic metabolites by occupants of moldy houses [27].

Among the most common health-related risks associated with molds and indoor dampness is the sick building syndrome (SBS) $[4,28]$. This syndrome has been associated with symptoms such as sensory irritation of the eyes, nose and throat, neurotoxic or general health problems, skin irritation, non-specific hypersensitivity reactions, and increasing cases of asthma [29]. A meta-analysis of 33 peer-reviewed studies found an increase in the relative risk of various adverse respiratory outcomes for individuals exposed to dampness and mold (Table 1) $[30,31]$.

Aside from moldy homes, the prevalence of indoor bioaerosols related to human health issues is also on the rise due to modern lifestyles in crowded, closed indoor spaces such as workplaces and schools [32]. Fungi (and bacteria) are found in bioaerosols of indoor environments. A range of fungal strains are considered of importance in indoor air samples, and various fungi have been identified in buildings, in particular black molds such as Stachybotrys chartarum and different Alternaria, Aspergillus, Cladosporium and Penicillium species. Species that are harmful and allergenic to humans, either directly or through their toxic metabolites, have been found to play a role in increasing the risk of developing atopic symptoms and allergic sensitization not only to molds, but to other commonly inhaled allergens as well [32-35].

In studies in several European countries, Canada and the US in 2004, at least $20 \%$ of buildings had one or more signs of dampness [3]. This estimate agrees with findings from a study of 16,190 people in Denmark, Estonia, Iceland, Norway and Sweden, which indicated an overall prevalence of

\begin{tabular}{|l|c|c|}
\hline \multicolumn{1}{|c|}{ Outcome } & No. of studies & Odds ratio (95\% Cl) \\
\hline Upper respiratory tract symptoms & 13 & $1.70(1.44-2.00)$ \\
\hline Coughing & 18 & $1.67(1.49-1.86)$ \\
\hline Wheezing & 22 & $1.50(1.38-1.64)$ \\
\hline Concurrent asthma & 10 & $1.56(1.30-1.86)$ \\
\hline Past diagnosis of asthma & 8 & $1.37(1.23-1.53)$ \\
\hline Asthma development & 4 & $1.34(0.86-2.10$ \\
\hline
\end{tabular}

Table 1: Summary of health risks from dampness and mold in US houses [31]. indoor dampness of $18 \%$, with the lowest prevalence in Gothenburg, Sweden (12.1\%) and the highest in Tartu, Estonia (31.6\%) [28]. Dampness was also more likely to occur in overcrowded houses that lack appropriate heating, ventilation and insulation [3].

\section{Primary Fungal Growth and High-Risk Groups}

Primary fungal growth on animal and human hosts produces diseases that are collectively termed mycoses. Mycoses are frequently acquired via inhalation of spores from an environmental reservoir or by unusual growth of a commensal species that normally resides on human skin or in the gastrointestinal tract [7]. These commensal species become pathogenic in the presence of antibacterial, chemotherapeutic or immunosuppressant drugs, human immunodeficiency virus infection, in-dwelling catheters and other predisposing factors [36,37]. Fungi that cause mycoses can be divided into two categories: primary pathogens (e.g. Coccidioides immitis and Histoplasma capsulatum) and opportunistic pathogens (e.g. Aspergillus fumigatus and Candida albicans). Primary pathogens affect otherwise healthy individuals with normal immune systems. Opportunistic pathogens produce illness by taking advantage of debilitated or immunocompromised hosts [7].

Aspergillus species most frequently cause infection in hospitalized patients who are markedly immunosuppressed; the typical host has neutropenia, is receiving corticosteroids, and often has undergone a transplant procedure and/or chemotherapy. In this setting, outbreaks of infection have been linked to construction work that results in high levels of contamination of the hospital environment [8]. Other, less invasive forms of pulmonary and upper respiratory/orbital aspergillosis can be found in older adults and are not acquired in the hospital; instead, they develop as a result of environmental exposure to these ubiquitous organisms [38].

Members of the genera Aspergillus, Fusarium and Mucorales are regarded as the main cause of fungal infections in hospitals [3941]. Prior to potential dissemination, spores emanating from these colonies can be inhaled by immunosuppressed patients, resulting in local infections [42]. Aspergillus fumigatus and A. flavus are the leading Aspergillus species causing invasive aspergillosis [43]. Outbreaks of hospital-acquired (nosocomial) aspergillosis infections are attributed to airborne sources and even small concentrations of spores have been associated with outbreaks [44].

For example, invasive aspergillosis is a major opportunistic infection in hematology patients. Spore inhalation is the usual route of Aspergillus infection, suggesting a determining role of environmental contamination by spores in the epidemiology of invasive aspergillosis. Alberti et al. [39] prospectively examined the relationship between environmental contamination by Aspergillus and other fungal species and the incidence of invasive nosocomial aspergillosis (INA) in a bone marrow transplant unit and two hematology wards. During a 4 year period, levels of air and surface fungal contamination were determined bimonthly in patients' rooms (some equipped with high-efficiency particulate air (HEPA) filters and laminar air flow (LAF) systems) and in various common areas in each ward (corridors, nursing stations, etc.). Results were compared to the incidence of INA. A total of 3100 air and 9800 surface samples were collected and 79 cases of invasive aspergillosis were diagnosed, 64 of which were probably or possibly INA. Patterns of fungal contamination were comparable in the three wards, with a gradient ranging from high levels in the common areas to a virtual absence in rooms equipped with a HEPA filter and LAF system. Using a regression model, a significant relationship was found between INA incidence and the degree of fungal contamination of air 
and surfaces in conventional patient rooms (not equipped with HEPA) and common areas [39].

\section{Toxic Fungal Secondary Metabolites}

\section{Mycotoxin pathogenicity}

Health-related risks associated with moldy and damp environments can be associated with primary fungal growth or with mycotoxins. However, human illnesses caused by mycotoxins may be a bigger than assumed public health problem because some of these cases (chronic) may go unnoticed for an extended period of time; only large amounts of consumed mycotoxins result in acute symptomology [45].

Mycotoxins are secondary metabolites produced by micro-fungi that can cause disease and death in humans and other animals. These metabolites produce diseases that are collectively termed mycotoxicoses [7]. Mycotoxicoses can result from skin contact with mold-infested substrates or inhalation of spore-borne toxins, both of which are important sources of exposure. Except for supportive therapy (e.g. diet, hydration), there are almost no treatments for mycotoxin exposure [7].

The symptoms of a mycotoxicosis depend on the type of mycotoxin, the amount and duration of the exposure, the age, health, and sex of the exposed individual and many other poorly understood synergistic effects involving genetics, dietary status, and interactions with other toxic insults. Thus, the severity of mycotoxin poisoning can be compounded by factors such as vitamin deficiency, caloric deprivation,

\begin{tabular}{|c|c|c|c|c|c|c|c|c|c|}
\hline \multirow[t]{2}{*}{ Fungal isolates } & \multirow{2}{*}{$\begin{array}{c}\text { No. of } \\
\text { tested } \\
\text { isolates }\end{array}$} & \multicolumn{2}{|c|}{ Protease } & \multicolumn{2}{|c|}{ Lipase } & \multicolumn{2}{|c|}{ Urease } & \multicolumn{2}{|c|}{ Hemolysis } \\
\hline & & + ve & -ve & +ve & -ve & +ve & -ve & +ve & -ve \\
\hline Aspergillus flavus & 20 & 16 & 4 & 19 & 1 & 20 & 0 & 19 & 1 \\
\hline Aspergillus fumigatus & 16 & 10 & 6 & 15 & 1 & 15 & 1 & 12 & 4 \\
\hline Aspergillus niger & 19 & 15 & 4 & 16 & 3 & 8 & 11 & 13 & 6 \\
\hline $\begin{array}{l}\text { Cladosporium } \\
\text { cladosporioides }\end{array}$ & 5 & 1 & 4 & 4 & 1 & 0 & 5 & 2 & 3 \\
\hline Fusarium solani & 17 & 11 & 6 & 9 & 8 & 10 & 7 & 7 & 10 \\
\hline Fusarium oxysporum & 6 & 3 & 3 & 5 & 1 & 5 & 1 & 4 & 2 \\
\hline Myrothecium roridum & 1 & 1 & 0 & 1 & 0 & 1 & 0 & 1 & 0 \\
\hline Stachybotrys elegans & 26 & 22 & 4 & 25 & 1 & 19 & 7 & 19 & 7 \\
\hline Total & 110 & 79 & 31 & 94 & 16 & 78 & 32 & 77 & 33 \\
\hline
\end{tabular}

Table 2: Extracellular enzymes produced by the tested fungal isolates collected from ICUs and ORs at Assiut University Hospital [6].

$+v e$ and -ve, positive and negative $\mathrm{pH}$ value, respectively, resulting from the fungal isolate by-products' further hydrolyzation alcohol abuse, and infectious disease status. In turn, mycotoxicoses can enhance vulnerability to microbial diseases, worsen the effects of malnutrition, and interact synergistically with other toxins [7].

Mycotoxins are an extremely diverse group of low-molecularweight fungal metabolites which, when ingested, inhaled or absorbed through the skin, can cause lowered performance, sickness or even death in man and animals, including birds [46,47]. Mycotoxins may affect the reproductive, immune and gastrointestinal systems, and specific target organs. In addition, they may affect hormonal activity and cause developmental defects, including those related to birth (teratogenic and neurotoxic) [48-50]. Although fungal spores may have the highest concentrations of mycotoxins, the vegetative part of the fungus, the mycelium or the substrate upon which the fungus grows can also contain these toxins. Viability of spores is not essential to toxicity. In other words, a dead spore can still be a source of toxin $[51,52]$.

\section{Extracellular enzyme pathogenicity}

Fungi are also known to secrete extracellular enzymes based on their growth substrate. Production and secretion of hydrolytic enzymes, such as proteases, lipases and phospholipases, are very important virulence factors. These enzymes play a role in nutrition, tissue damage, fungal dissemination within the human body, iron acquisition and overcoming the host immune system, all of which strongly affect fungal pathogenicity [53]. Secretion of enzymes into extracellular environments might be an important adaptive mechanism during the fungal life cycle [54]. It is logical to suppose that these enzymes act by facilitating tissue invasion, but they can also participate in causing infection by impairing some mechanisms of the immune system and/or assisting in obtaining nutrients, thus causing injury to the host $[55,56]$.

To enable a more accurate identification and prediction of hospitalacquired fungal outbreaks, including exposure to their extracellular enzymes and mycotoxins, a total of 110 isolates belonging to 8 fungal species collected from intensive care units (ICUs) and operating rooms (ORs) at Assiut University Hospital in Egypt were examined for their ability to produce extracellular enzymes and mycotoxins that are considered important in fungal pathogenicity [6]. El-Kady and Moubasher [57] showed that 73, 92 and 78 of the 110 tested isolates produced protease, lipase and urease, respectively. Moreover, 77 of the tested isolates exhibited hemolytic activity (Table 2). Using thinlayer chromatography to screen for mycotoxins in extracts of the tested fungal isolates, El-Kady and Moubasher [57] revealed that 79 isolates had the ability to produce at least one of these mycotoxic compounds (aflatoxins B1, B2, G1, etc.; Table 3). These results demonstrated that

\begin{tabular}{|c|c|c|c|}
\hline Toxins produced & No. of strains positive & No. of strains tested & Fungal isolates \\
\hline Aflatoxins B1, B2 & 10 & \multirow{2}{*}{20} & \multirow{2}{*}{ Aspergillus flavus } \\
\hline Aflatoxins B1, B2, G1, G2 & 1 & & \\
\hline Gliotoxin, fumagillin & 16 & 16 & Aspergillus fumigatus \\
\hline Gliotoxin & 7 & 19 & Aspergillus niger \\
\hline Cladosporin & 5 & 5 & Cladosporium cladosporioides \\
\hline T-2 toxin & 9 & \multirow{3}{*}{17} & \multirow{3}{*}{ Fusarium solani } \\
\hline T-2 toxin, zearalenone & 2 & & \\
\hline Zearalenone & 1 & & \\
\hline Zearalenone & 6 & 6 & Fusarium oxysporum \\
\hline Roridin $\mathrm{A}$ and $\mathrm{E}$, Verrucarin $\mathrm{A}$ and $\mathrm{J}$ & 1 & 1 & Myrothecium roridum \\
\hline Trichoveroides & 10 & \multirow{2}{*}{26} & \multirow{2}{*}{ Stachybotrys elegans } \\
\hline Trichoveroides, Verrucarin $\mathrm{J}$ & 6 & & \\
\hline Satratoxin H\&E, Trichoveroids, Verrucarin J & 5 & & \\
\hline
\end{tabular}

Table 3: Mycotoxigenicity of fungal isolates collected from ICUs and ORs at Assiut University Hospital [6]. 
the opportunistic fungal species isolated from ICUs and ORs exhibit enzymatic and mycotoxic activities that are most effective virulence factors in fungal pathogenicity.

\section{Fungal Pathogenicity and Mycotoxigenicity in High- Risk Groups in the General Population}

It is not only hospitalized and immunosuppressive patients who are susceptible to fungal species pathogenicity and mycotoxigenicity. There are other high-risk groups in the general population, including children and the elderly. For example, the level of adverse health effects was significantly higher in children in Italy, Denmark, Sweden, Norway and France studying in schools that had high mold/dampness levels. Dry cough at night and rhinitis were the most commonly reported symptoms. Viable indoor molds and total fungal DNA were commonly found in the monitored European schools and were adversely related to respiratory health [10]. In Malaysia, data on the prevalence of respiratory symptoms and infections among pupils aged 14-16 years were examined, in an attempt to associate environmental allergens, fungal DNA and mycotoxin exposure with health problems. In particular, the Malaysian team showed a positive association between levels of Aspergillus versicolor DNA and wheezing and daytime attacks of breathlessness, whereas Streptomyces DNA was positively associated with medically diagnosed asthma [21]. In a small-scale population study in Israel, including 395 members of one rural community, the rate of positive skin tests for at least one of the fungal allergens tested was reported to be $30.5 \%$ in the age range of $4-9$ years [58]. Infants and children are considered more susceptible to different toxins than adults because of their lower body weight, higher metabolic rate, and incomplete development of some organs and tissues, such as those of the central nervous system [11,59].

Clinical aspects of fungal infections in older adults include endemic mycoses. Among these, histoplasmosis is unique in that several manifestations of infection are found almost entirely in the elderly [9]. People can get histoplasmosis after breathing in airborne microscopic fungal spores. Many people who contract histoplasmosis will get better on their own without medication, but in some people, such as those who have weakened immune systems, the infection can become severe [60]. Chronic cavitary pulmonary histoplasmosis occurs, almost without exception, in older men who have emphysema. Infection progress seems to be inexorable, because the host is unable to eradicate the organism from severely emphysematous lungs [61]. The second form of histoplasmosis that is characteristically found in older adults is chronic progressive disseminated histoplasmosis. Patients with this disease manifestation are not overtly immunosuppressed but probably have a selective defect in cell-mediated immunity to Histoplasma capsulatum. The disease is characterized by overwhelming parasitization of the reticuloendothelial system. Macrophages in the lymph nodes, liver, spleen, bone marrow and lungs contain large numbers of yeast [62].

Infections with opportunistic fungi are on the rise because today, older patients are more likely to be considered for transplants, aggressive regimens of chemotherapy for cancer and administration of immunosuppressive drugs for nonmalignant diseases. Older individuals are more likely than younger ones to develop complications when infected with Coccidioides immitis. This may be because of agerelated changes in the immune system or because of concomitant diseases, such as diabetes mellitus, which is a risk factor for more severe pulmonary coccidioidomycosis [63].

\section{Targeted and Proportionate Approaches for Identifying and Monitoring Primary or Secondary Fungal Loads}

To determine where more refined techniques for qualitative identification and quantitative monitoring of diverse fungal species or their mycotoxins may be needed, the advantages and limitations of these diverse techniques must be reviewed and compared. Once these are recognized, their applicability and cost effectiveness relative to health-related mycological symptoms and pathogenicity in certain target locations or risk groups can be discussed. The threshold for EIs and the sensitivities of the diverse techniques used for qualitatively or quantitatively assessing mycological load also need to be considered.

Environmental Inspections should be performed whenever a mold problem is suspected. Air handling units and building areas subject to condensation or water damage should be examined for visible mold growth. The presence of mold growth is sufficient reason to undertake remedial measures, regardless of the species found [64]. In the US, for example, guidelines for the assessment and remediation of fungi in indoor environments include recommendations for visual inspection to identify possible mold problems, environmental sampling to confirm the presence of visually identified mold, moisture control, cleaning and building repairs as well as guidelines for the protection and training of workers engaged in maintenance and remediation work associated with mold [65].

To determine indoor airborne fungal loads, an integration of methods should be considered. These can include EI and long-term sampling, e.g. of settled dust or pumped air, and use of total fungal analyses, such as for ergosterol, beta 1-3 glucan or total molds by the CAMERANEA method [21-23] or fungal DNA analysis via quantitative or qualitative polymerase chain reaction (PCR) [21-24].

Samples are sometimes collected from the air or surfaces during building assessments, and fungal spore levels and types are evaluated using noncultured-via air sampling, or cultured-via Petri dish methodologies, as a surrogate measure of exposure to fungal allergens [19]. The simplest method for evaluation of airborne fungal spore levels is the OPD, based on gravitational settling of fungal spores on an agar medium. Although only viable and heavy fungal units are measured with the OPD method, it is frequently used because it is inexpensive. Nevertheless, OPD sampling for viable fungi has severe limitations and is influenced by many external factors (e.g. wind velocity outdoors, among others). Moreover, small spores of Penicillium and Aspergillus will not settle on Petri dishes because they are too light; they must be pulled down onto the OPD by an air sampler, such as the Andersen sampler.

More advanced methods include active air sampling [20], supporting the measurement of a wider range of airborne fungal types and covering a greater air volume than the OPD method. Moreover, microscopic and DNA analyses of total fungal units accumulated via automatic air sampling may result in higher fungal levels since these measure viable and nonviable fungal units. Air sampling for microbiological monitoring (such as with the MAS-100 Merck) can also be used. However, some have argued that air sampling is not recommended unless fungal growth is observed or occupant complaints are serious [64].

The advantage of nonviable over viable fungal air sampling is that detection of fungal structures (spores, hyphal fragments, etc.) is not dependent on their viability or suitability of the agar media. Nonviable air samples are collected with samplers such as Air-O-Cell, Allergenco, 
Burkard, Cyclex, Cyclex-d and Micro-5, among others. The spores (viable or dead) and other particulates are trapped on the sticky surface of the spore trap and can then be directly enumerated and identified under a microscope. Viable air samples are often collected on agar media either on strips (for the Reuter centrifugal air sampler) or on Petri dishes (for the Andersen sampler). Unlike nonviable air sampling, detection and subsequent enumeration and identification of airborne fungal particulates collected on growth media depends on whether the spores and hyphal fragments are viable and whether the media used can support their growth into colonies. For this reason, colony counts are usually lower than spore counts. Even if all of the fungal structures are viable, colony counts are likely to be lower than the spore/hyphal fragment counts because what is counted as a single colony may have developed from more than a single spore or hyphal fragment. Since both nonviable and viable air sampling has limitations, use of either method alone is inadequate. To obtain conclusive information on the level of contamination and the diversity of airborne fungi in a building, taking both viable and nonviable air samples is preferable (http://www. moldbacteriaconsulting.com/fungi/non-viable-fungal-air-samplingalone.html after [66].

Once fungal sampling has been completed, fungal community structure must be specifically screened and identified, both qualitatively and quantitatively. Indoor fungi are traditionally studied by plate cultivation and nearly all of the information on their occurrence and prevalence relies on viability studies. However, the restrictions of cultivation are known to bias the picture of fungal community structure both qualitatively and quantitatively [67]. Medium composition for the enumeration of viable fungal units is important and might influence the environmental fungal species that are cultured and identified [68]. Moreover, the number, dispersion potential and survival of fungal spores vary among species [69]. Some organisms can only be identified at the genus or group level and some may remain morphologically indistinguishable under laboratory conditions. The main benefits of using DNA as an identification target instead of cultivation-based methods are the speed, accuracy and analytical sensitivity of detection and the ability to detect and identify dead or dormant organisms $[70,71]$.

The molecular methods that are most often used in fungal studies include conventional (qualitative) PCR, involving molecular identification of species only, quantitative PCR (qPCR), involving quantitative data on the identified fungal species or groups [7275], universal fungal PCR combined with denaturing-gradient gel electrophoresis (DGGE), temperature-gradient gel electrophoresis [76-78], terminal or conventional restriction fragment length polymorphism analysis [72,79], next-generation DNA sequencing (NGS) alone or combined with qPCR [80] and simple labeling-based fingerprinting methods [81]. Using $\mathrm{qPCR}$ has provided valuable information on the occurrence of the most common indoor fungi and this method shows great promise for the rapid provision of quantitative data on the occurrence of the studied organisms [82]. However, apart from cloning and sequencing of amplified fungal DNA, or in some cases DGGE followed by sequencing, these methods do not produce detailed information on previously unknown or unexpected taxa [24]. A comparison of methods has suggested that the cloning, cultivation and qPCR methods complement each other, generating a more comprehensive picture of fungal flora than any of the methods used alone [24].

Among the important determinants of the physical processes that act upon airborne fungi is their aerodynamic diameter. These physical processes include gravitational settling, respiratory deposition, penetration into buildings, resuspension from surfaces into air and long-range transport. To evaluate diverse, taxon-specific, fungal aerodynamic diameters from bioaerosol samples, Yamamoto et al. [80] combined NGS with qPCR. The accuracy of the combined method was demonstrated by comparing geometric mean aerodynamic diameters of selected taxa produced by the NGS-based method to the diameters produced by taxon-specific qPCR $(r=0.996)$. The results reported in their study demonstrated the robust nature of this method, providing novel insights into the aerodynamic properties of diverse airborne fungal species and potentially enabling a better accounting of taxonspecific fungal fate and exposure in both indoor air and the atmosphere [80].

\section{Methods to Measure Fungal Toxins/Mycotoxins}

Because fungal viability may be short-lived compared to toxin stability, methods of detecting toxins or toxicity are much preferred over those requiring fungal culturing. Public health decisions would be greatly facilitated by the development of rapid and affordable strategies that provide an accurate quantitative assessment of possible environmental exposure to fungal toxins [83]. In the past, microbial investigations of indoor air and exposure assessments generally relied on mycological identification using viable and nonviable methods (culture identification, fungal spore counts, bulk and air sampling), which may give qualitative or quantitative information about the presence of particular fungi, but not their particular properties or toxicity [84]. In Bloom et al.'s [85] study, high-performance liquid chromatography (HPLC) and mass spectrometry (MS), especially tandem MS involving multiple steps of MS selection with some form of fragmentation between stages (MSMS), providing high analytical specificity, and gas chromatography (GC) proved to be complementary tools for detecting some of the most potent mycotoxins produced by molds that are frequently encountered in damp indoor environments. Combined GC and MSMS was used to determine the amounts of verrucarol and trichodermol and their respective hydrolysis products macrocyclic trichothecenes and trichodermin, of Stachybotrys chartarum, while HPLC with MSMS was used to determine the amounts of satratoxin $\mathrm{G}$ and satratoxin $\mathrm{H}$, which are cytotoxic mycotoxins produced by $S$. chartarum, citrinin, gliotoxin, and patulin produced by, for example, Aspergillus spp. and Penicillium spp. and sterigmatocystin, a carcinogenic mycotoxin produced mainly by Aspergillus versicolor, in samples from water-damaged indoor environments [85]. The latter three mycotoxins have been shown to be immunomodulatory, causing polarization in cytokine production toward a Th2 phenotype [86] and citrinin causes depletion of intracellular glutathione at nontoxic concentrations [87].

Indoor molds may fragment into very small airborne mycotoxincontaining particles, resulting in an up to 500-fold higher exposure than previously assumed [13,88-90]. In addition, Cho et al. [91] showed that the respiratory deposition of $S$. chartarum fragments is over 200fold higher than that of spores in adults and an additional 4 to 5 times higher in infants. These aerosolized fragments could potentially also be a source of allergens [92].

\section{Evaluating the Impact of Fungal Load Reduction}

In general, there is no gold standard for the expected response rate in terms of fungal load reduction and its clinical significance based on a comparison of treated and untreated moldy and damp environments and the reduction rate is based on a diverse range. Moreover, no universally agreed upon standard exists for acceptable 
fungal load. However, a current set of local guidelines is available for indoor fungal levels published by different organizations in different countries, with greatly varying ranges of acceptable concentrations, as summarized by Giardino [17]. For example, The American Conference of Governmental Industrial Hygienists [18] suggested that fewer than $100 \mathrm{CFU} / \mathrm{m}^{3}$ is considered negligible, while $200 \mathrm{CFU} / \mathrm{m}^{3}$ is recommended as a guideline for indoor airborne fungi.

While special attention has been given in some countries to fungal contamination and remediation in schools and commercial buildings, including published guidelines to prevent mold risk; the United States Environmental Protection Agency (EPA) [93], no significant progress has been made in translating the fungal risk base into an environmental health policy that would include guidelines for monitoring fungal load and the associated health burden. The significance of fungusrelated risks has led some European countries and the US $[19,65,93]$ to recognize the need for guidelines for improving indoor environmental quality with respect to fungal load and fungus-related chemicals. Doseresponse relationships between exposure to fungi and symptoms are lacking. A number of numerical standards for "acceptable" levels of indoor fungi have been proposed $[17,94]$.

In one environmental study, for example, following remediation actions, the total amounts of airborne mold spores measured in 23 dwellings had decreased. In 11 dwellings, the number was reduced to $1 / 10^{\text {th }}$ and in 7 dwellings to $1 / 5^{\text {th }}$ of the levels at first sampling. Only one dwelling showed no reduction. Thus, a significant reduction (5-10-fold) in fungal load was observed, equal to a reduction rate of $80-90 \%$ in mycological load [95]. In another environmental study, Meklin et al. [82] demonstrated the effects of renovating a moistureand mold-damaged school building on the schoolchildren's health and exposure. Microbial sampling from indoor air at the school and a health questionnaire study were performed before and after the renovation. The results were compared to those from an undamaged reference school. The effect of a thorough renovation in the damaged school was reflected in decreased concentrations of airborne fungi and decreased diversity of mycoflora. There was a significant decrease in the prevalence of respiratory symptoms among the schoolchildren after the renovation. The results showed that the symptoms were associated with moisture damage, and that the increasing symptom levels could be normalized with proper repair measures [82]. In the further cross-sectional study, the questionnaires provided information on the respiratory symptoms and health of the participating children. Children aged 7 to 13 years participated in the study before and after the intervention, respectively. Numbers of participating children at the beginning and end of the study were 414/408 and 431/296 in damaged and nondamaged schools, respectively. The questionnaire contained 32 questions concerning, among others, personal characteristics, home environment, perceived indoor air-quality characteristics and occurrence of respiratory symptoms. Symptom manifestations during the fall and spring terms were referred to separately [82]. Kercsmar et al. [96] also showed that home remediation aimed at moisture sources results in a reduction in asthma morbidity in children. Another important approach recommended by the New York City Department of Health and Mental Hygiene [65] was implementation of a round of communications with occupants of affected spaces, especially when mold growth required large-scale remediation.

Nevertheless, assessing the impact of EIs and the associated corrective measures may be limited, and a means to overcome the reporting differences found in many studies comparing the occupants' reports on dampness to external expert inspections $[1,97,98]$ needs to be addressed; moreover, local staff carrying out the inspections, or individuals occupying the schools, including the teachers and parents, need to be consulted while completing the questionnaires. Notifications from occupants regarding their indoor environmental complaints could include complaints of discomfort (noise, temperature, odors, etc.) and specific areas in schools or rooms [99].

Reporting differences have been found in many studies comparing occupants' reports on dampness to external expert inspections, with the occupants reporting more dampness than the trained surveyors $[2,98,100]$. Those studies found that occupants' reports of damp or fungal spots correlated better with an objective measure of indoor fungi than investigators' reports of these visible signs; a similar finding was reported by Sun et al. [98]. Looking for more objective measures to validate occupants' health complaints regarding the extent of mold exposure, Dales et al. [101] argued that reported residential fungal contamination is consistently associated with increased symptoms among occupants; however, an objective measure of health effects is lacking and a pathophysiological mechanism has not been established.

Dales et al. [101] set out to determine whether exposure to indoor fungal contamination influences T-cell differentiation. In that study, they contrasted lymphocyte populations, measured by flow cytometry, in a group of children who lived in homes with considerable fungal contamination to those in a group living in less contaminated homes [101]. Living in a more contaminated versus less contaminated house was associated with a larger number of $\mathrm{CD} 3+\mathrm{T}$ cells expressing $\mathrm{CD} 45 \mathrm{RO}\left(1.5 \times 10^{9} / \mathrm{L}\right.$ versus $1.1 \times 10^{9} / \mathrm{L}$, respectively, $p=0.05$, two-tailed $\mathrm{t}$ testing) and a reduced CD4/CD8 ratio (1.6 versus 1.8 , respectively, $p=0.04$ ). The differences persisted over a 12 month period and they were not explained by the child's age or total serum IgE, dust mite antigens or the presence of furry or feathered pets or a humidifier. The results suggested that residential fungal contamination leads to chronic stimulation of children's lymphocytes [101].

Looking for more objective measures to validate/compare the occupants' subjective complaints and the results of external expert inspections, the fractional exhaled nitric oxide (FeNO) test can serve as an objective surrogate measure for inflammatory respiratory burden [102,103]. This is a simple noninvasive method of assessing inflammation in asthma and it is now well established that FeNO is the first bedside test to indirectly assess eosinophilic airway inflammation in the bronchial mucosa in asthmatics [104]. Seeking a reliable correlation between indoor mold exposure and asthma, Vesper and Wymer [105] turned the qualitative link between indoor mold exposure and asthma, which has been assumed for over 25 years, into a quantitative assessment of asthma risk from mold exposure as estimated by the home's environmental relative moldiness index (ERMI) value. The home's ERMI value was derived from qPCR analysis of 36 molds in a dust sample. Six epidemiological studies of the relationship between ERMI values and asthma, in cities across the US, showed that both children and adults with asthma were living in homes with significantly higher ERMI values than the control or comparison homes [105].

Recently, Norbäck et al. [22] studied the associations between ocular symptoms, rhinitis, throat and dermal symptoms, headache and fatigue in students and exposure to chemical microbial markers and fungal DNA in vacuumed dust in Malaysian schools. Dust was vacuumed and analyzed for levels of five types of endotoxin as 3-hydroxy fatty acids (C10, C12, C14, C16 and C18 3-OH), muramic acid, ergosterol and five sequences of fungal DNA. There were positive associations between the amount of fine dust in the dust samples and ocular symptoms $(p<0.001)$ and rhinitis $(p=0.006)$. There were positive associations between $\mathrm{C} 14$ 
3-OH and rhinitis $(p<0.001)$ and between $\mathrm{C} 18$ 3-OH and dermal symptoms $(p=0.007)$. There were negative (protective) associations between levels of total endotoxin (lipopolysaccharide) $(p=0.004)$ and levels of ergosterol $(p=0.03)$ and rhinitis, and between $\mathrm{C} 123-\mathrm{OH}$ and throat symptoms $(p=0.004)$. Overall, the amount of fine dust in the classroom was associated with rhinitis and other SBS symptoms, and improved cleaning of the schools was therefore deemed important. Endotoxin in the school dust seemed to be mainly protective for rhinitis and throat symptoms, but different types of endotoxin could have different effects [22].

Despite the potential health risks associated with fungal units (spores) and fungal-related toxins, there is no consensus on a fungusor mycotoxin-specific identification approach, nor is there any agreedupon universal standardized or validated approach for monitoring and mitigating the overall mycological load in contaminated environments. Similarly, there is no gold standard for an acceptable reduction rate of mycological contamination following diverse monitoring and mitigation strategies. The severity of the response and the health-related symptomology of an individual's exposure to fungal spores or their mycotoxins depend on the type of exposure, the amount and duration of the exposure, the age, health and sex of the exposed individual and many poorly understood synergistic effects involving genetics, dietary status, and interactions with other toxic insults [7].

Some methodologies restrict their environmental monitoring to measuring culturable and viable mold due to the higher complexity and/or cost of DNA analyses, which measure nonviable molds as well. Some methodologies go beyond the fungal spore-borne toxin counts by determining indoor mold fragments in very small, airborne, mycotoxin-containing particles, resulting in an up to 500-fold higher exposure level than previously assumed [13,88-90].

Regardless, the degree of environmental monitoring and the level of refinement of the methodology chosen to identify and quantify the specific fungi and their mycotoxins should be weighed against the level of risk associated with the mycological health symptomology, mycotoxigenicity and pathogenicity of the fungal isolates. As such, we should be able to evaluate whether using an integrated array of monitoring methods, including EI, OPD and airborne sampling with or without molecular analysis methods (conventional or qPCR specific for fungal species or groups or universal fungal PCR) is valuable as a monitoring protocol for identifying the levels and sources of fungal load. The use and cost effectiveness of extracting fungal isolates and mycotoxic compounds should be considered where strict surveillance is required in high-risk environments such as ICUs, ORs, bone marrow transplant units and hematology wards or when high-risk groups are involved, i.e., children and the elderly.

Patients' hospital rooms can also be equipped with HEPA filters and LAF systems to control the levels of air and surface fungal contamination. However, as primary fungal pathogens may just as easily affect otherwise healthy individuals with normal immune systems [7], the diverse monitoring methods for identification and quantification of these primary fungi in 'normal' building environments should be considered as well. In addition, remediation activities should conclude with a determination of whether the mold has been completely eradicated, including a professional judgment of whether the cleanup was sufficient. Further, the site should be revisited shortly after remediation to ascertain that visible mold, mold-damaged materials and moldy odors are no longer present, and that there are no signs of water damage or mold growth [93].
Ultimately, it is the future of a growing evidence base that will lay the groundwork for public policies and guidelines worldwide for both monitoring and preventing moisture and mold problems in buildings and mitigating them when they do occur. Wanless report states that "every opportunity to generate evidence from current policy and practice" should be taken to provide more robust evidence of practical interventions that might help governments formulate effective policies [106]. Evidence of higher individual risk does not specifically address the primary causal agents responsible for the reported health outcomes. No one expects, for example, that dampness per se is a causal agent, but dampness (or moisture) is known to promote the growth and proliferation of dust mites, mold and bacteria, exposure to which can result in allergic or infectious health outcomes. Whatever the primary causal agents, policies and programs that are successful in monitoring, preventing and mitigating dampness and mold conditions will also be effective at reducing public health risks and their associated economic impacts [4].

\section{Conclusion and Limitations}

To enable a more accurate qualitative identification and quantification of the specific mycological load, including fungal units (spores) and fungal related toxins, the use of an array of monitoring methods needs to be assessed. The efficacy and cost-effectiveness of those monitoring methods separately or in combination can be compared based on their ability to measure culturable and viable mold via air sampling or measure non-viable mold by using, for example, DNA analyses, or using methodologies which go beyond the fungal spore-borne toxin counts by determining indoor mold fragments in very small, airborne, mycotoxin-containing particles. Overall the efficacy and cost effectiveness of those monitoring and identification methods can be compared against the relative expected reduction rate of mycological contamination following diverse monitoring and mitigation strategies. The expected reduction rate and level of refined monitoring and mitigation techniques are relative to the population at risk, type of exposure and other toxic interactions in the environment.

\section{References}

1. Bornehag CG, Sundell J, Bonini S, Custovic A, Malmberg P, et al. (2001) Dampness in buildings and health. Nordic interdisciplinary review of the scientific evidence on associations between exposure to dampness and health effects. NORDDAMP. Indoor Air 11: 72-86.

2. Bornehag CG, Blomquist G, Gyntelberg F, Jarvholm B, Malmberg P (2004) Dampness in buildings as a risk factor for health effects, EUROPO. A multidisciplinary review of the literature (1998-2000) on dampness and mite exposure in buildings and health effects. Indoor Air 14: 243-257.

3. Institute of Medicine (IOM) (2004) Board on health promotion and disease prevention, committee on damp indoor spaces and health. Damp indoor spaces and health. National Academies Press, Washington, DC.

4. Mudarri D, Fisk WJ (2007) Public health and economic impact of dampness and mold. Indoor Air 17: 226-235.

5. Atalla A, Garnica M, Maiolino A, Nucci M (2015) Risk factors for invasive mold diseases in allogeneic hematopoietic cell transplant recipients. Transplant Infectious Disease 17: 7-13.

6. Aboul-Nasr MB, Ahmed-Zohari AN, Amer EM (2013) Enzymatic and toxigenic ability of opportunistic fungi contaminating intensive care units and operation rooms at Assiut University Hospitals, Egypt. Springer Plus 2: 1-6.

7. Bennett JW, Klich M (2003) Mycotoxins. Clin Microbiol Rev16: 497-516.

8. VandenBergh MFQ, Verweij PE, Voss A (1999) Epidemiology of nosocomial fungal infections: Invasive aspergillosis and the environment. Diagn Microbiol Infect Dis 34: 221-227.

9. Kauffman CA (1995) Endemic mycoses in older adults. Infect Dis Clin Pract 4: 41-45. 
Citation: Masaphy S, Ezra R (2016) Targeted Inspection of Environmental Mycological Load for Mitigation of Indoor Mold toward Improved Public Health. J Microb Biochem Technol 8: 449-458. doi: 10.4172/1948-5948.1000324

10. Simoni M, Cai GH, Norback D, Annesi-Maesano I, Lavaud F et al. (2011) Total viable molds and fungal DNA in classrooms and association with respiratory health and pulmonary function of European schoolchildren. Pediatr Allergy Immunol 22: 843-852.

11. World Health Organization (WHO) (Environmental Health Criteria) (1986) Principles for evaluating health risks from chemicals during infancy and early childhood; the need for a special approach. IPCS, Geneva.

12. Buiarelli F, Di-Filippo P, Riccardi C, Pomata D, Rumolo E, et al. (2015) Analytical method for the determination of mycotoxins in indoor/outdoor airborne particulate matter by HPLC-MS-MS. Int J Environ Analyt Chem 95: 713-729.

13. Brasel TL, Martin JM, Carriker CG, Wilson SC, Straus DC (2005) Detection of airborne Stachybotrys chartarum macrocyclic trichothecene mycotoxins in the indoor environment. Appl Environ Microbiol 71: 7376-7388.

14. Bünger J, Westphal G, Mönnich A, Hinnendahl B, Hallier E, et al. (2004) Cytotoxicity of occupationally and environmentally relevant mycotoxins. Toxicol 202: 199-211.

15. Huttunen K, Pelkonen J, Nielsen KF, Nuutinen U, Jussila J, et al. (2004) Synergistic interaction in simultaneous exposure to Streptomyces californicus and Stachybotrys chartarum. Environ. Health Perspect 112: 659- 665.

16. Wang Y, Chai T, Lu G, Quan C, Duan H et al. (2008) Simultaneous detection of airborne aflatoxin, ochratoxin and zearalenone in a poultry house by immunoaffinity clean-up and high-performance liquid chromatography. Environ Res 107: 139-144.

17. Giardino NJ (2004) Summary of currently available guidelines for fungal levels in indoor spaces. IEQ Review.

18. American Conference of Governmental Industrial Hygienists (ACGIH) (1989) Guidelines for the assessment of bioaerosols in the indoor environment, Cincinnati, Ohio.

19. World Health Organization (WHO) (2009) Dampness and mold: WHO guidelines for indoor air quality: Dampness and mold.

20. Pasquarella C, Pitzurra O, Savino A (2000) The index of microbial air contamination. Journal of Hospital Infection 46: 241-256.

21. Cai GH, Hashim JH, Hashim Z, Ali F, Bloom E, et al. (2011) Fungal DNA allergens, mycotoxins and associations with asthmatic symptoms among pupils in schools from Johor Bahru, Malaysia. Pediatr Allergy Immunol 22: 290-297.

22. Norbäck D, Hashim JH, Markowicz P, Cai GH, Hashim Z, et al. (2016) Endotoxin, ergosterol, muramic acid and fungal DNA in dust from schools in Johor Bahru, Malaysia-Associations with rhinitis and sick building syndrome (SBS) in junior high school students. Sci Total Environ 545: 95-103.

23. Palmgren UGGP, Ström G, Blomquist G, Malmberg P (1986) Collection of airborne micro-organisms on Nuclepore filters, estimation and analysisCAMNEA method. J Appl. Bacteriol 61: 401-406.

24. Pitkäranta M, Meklin T, Hyvärinen A, Paulin L, Auvinen P et al. (2008) Analysis of fungal flora in indoor dust by ribosomal DNA sequence analysis, quantitative PCR and culture. Appl Environ Microbiol 74: 233-244.

25. Creasia DA, Thurman JD, Jones LJ (1987) Acute inhalation toxicity of T-2 mycotoxin in mice. Fundam Appl Toxicol 8: 230-235.

26. Smith JE, Anderson JG, Lewis CW, Murad YM (1992) Cytotoxic fungal spores in the indoor atmosphere of the damp domestic environment. FEMS Microbiol Lett 100: 337-343.

27. Polizzi V, Delmulle B, Adams A, Moretti A, Susca A, et al. (2009) JEM Spotlight: Fungi, mycotoxins and microbial volatile organic compounds in mouldy interiors from water-damaged buildings. J Environ Monit 11: 1849-1858.

28. Gunnbjornsdottir MI, Franklin KA, Norback D, Bjornsson E, Gislason D, et al (2006) Prevalence and incidence of respiratory symptoms in relation to indoor dampness: the RHINE study. Thorax 61: 221-225.

29. Godish T (2001) Indoor environmental quality. CRC Press, New York.

30. Fisk WJ, Eliseeva EA, Mendell MJ (2010) Association of residential dampness and mold with respiratory tract infections and bronchitis: A meta-analysis. Environ Health 9: 72

31. Fisk WJ, Lei-Gomez Q, Mendell MJ (2007) Meta-analyses of the associations of respiratory health effects with dampness and mold in homes. Indoor Air 17: 284-295.
32. Manda J, Helmut B (2011) Bioaerosols in Indoor environment - a review with special reference to residential and occupational locations. Open Environ. Bio Monit J 4: 83-96.

33. Hedayati MT, Pasqualotto AC, Warn PA, Bowyer P, Denning DW (2007) Aspergillus flavus: Human pathogen, allergen and mycotoxin producer. Microbiol 153: 1677-1692.

34. Kuhn DM, Ghannoum MA (2003) Indoor mold, toxigenic fungi and Stachybotrys chartarum: Infectious disease perspective. Clin Microbiol Rev 16: 144-172.

35. Schwab CJ, Straus DC (2004) The roles of Penicillium and Aspergillus in sick building syndrome. Adv Appl Microbiol 55: 215-238.

36. Calderone RA, Cihlar RL (2002) Fungal pathogenesis: Principles and clinical applications. Marcel Dekker, Inc., New York.

37. Dixon DM, McNeil MM, Cohen ML, Gellin BG, LaMontagne JR (1996) Fungal infections. A growing threat. Public Health Rep 111: 226-235.

38. Daly AL, Bradley SF, Velaquez LA, Kauffman C (1989) Mucormycosis: Association with deferoxamine therapy. Am J Med 87: 468-471.

39. Alberti C, Bouakline A, Ribaud P, Lacroix C, Rousselot P, Leblanc T (2001) Relationship between environmental fungal contamination and the incidence of invasive aspergillosis in haematology patients. J Hosp Infect 48: 198-206.

40. Faure O, Fricker-Hidalgo H, Lebeau B, Mallaret MR, Ambroise-Thomas P, et al. (2000) Eight year surveillance of environmental fungal contamination in hospital operating rooms and haematologic units. J Hosp Infect 50: 155-160.

41. Perdelli F, Cristina ML, Sartini M, Spagnolo AM, Dallera M, et al. (2006) Fungal contamination in hospital environments. J Infect Cont Hosp Epidemiol 27: 44-47.

42. Singh N, Paterson DL (2005) Aspergillus infections in transplant recipients Review. J Clin Microbiol 18: 44-69.

43. Pasqualotto AC (2008) Differences in pathogenicity and clinical syndromes due to Aspergillus fumigatus and Aspergillus flavus. J Med Mycol 47:1-10.

44. Vonberg RP, Gastmeier P (2006) Nosocomial aspergillosis in outbreak settings. J. Hosp Infect 63: 246-254

45. Hesseltine CW (1985) Global significance of mycotoxins. In J Mycotox and Phyco-toxins. Edited by: Steyn PS, Vleggaar R. Elsevier Scientific Publishing Co., Amsterdam.

46. Pitt Jl (1996) What are mycotoxins? Austral Mycotoxin Newsletter 7: 1-3

47. Van Egmond HP, Speijers GA (1999) Natural toxins I. Mycotoxins. In: van de Heijden K, Younes M, Fishbone L, Miller S, (Eds): International Food Safety Handbook, Science, International Regulation and Control. Marcel Dekker, Inc. New York, Basel.

48. Richard J (1991) Mycotoxins as immunemodulators in animal systems. In Mycotoxins, Cancer and Health. In: Bray GA, Ryan DH (Eds), Pennington Center Nutrition Series. Louisiana State University Press, Baton Rouge.

49. Sharma RP (1993) Immunotoxicity of mycotoxins. J Dairy Sci 76: 892-897.

50. Kuiper-Goodman T (2004) Risk assessment and risk management of mycotoxins in food. In: Mogan N, Olsen M (Eds), Mycotoxins in Food, Detection and Control. CRC Press, New York.

51. Keller NP, Turner G, Bennett JW (2005) Fungal secondary metabolism - from biochemistry to genomics. J Nat Rev Microbiol 3: 937-947.

52. Gauthier T, Wang X, Dos Santos JS, Fysikopoulos A, Tadrist S, et al. (2012 Trypacidin, a spore-borne toxin from Aspergillus fumigatus, is cytotoxic to lung cells. PloS One 7: 29906.

53. Ibrahim AS, Mirbod F, Filler SG, Banno Y, Cole GT, et al. (1995) Evidence implicating phospholipase as a virulence factor of Candida albicans. J Infect Immun 63: 1993-1998.

54. Monod M, Capoccia S, Lechenne B (2002) Secreted proteases from pathogenic fungi. Int Med J Microbiol 292: 405-419.

55. Birch M, Denning DW, Robson GD (2004) Comparison of extracellular phospholipase activities in clinical and environmental Aspergillus fumigatus isolates. J Med Mycol 42: 81-86.

56. Da Silva BCM, Auler ME, Ruiz LS (2005) Trichophyton rubrum isolated from AIDS and human immunodeficiency virus-infected patients in São Paulo, Brazil Antifungal susceptibility and extracellular enzyme production. J Chemotherapy 51: $21-26$. 
Citation: Masaphy S, Ezra R (2016) Targeted Inspection of Environmental Mycological Load for Mitigation of Indoor Mold toward Improved Public Health. J Microb Biochem Technol 8: 449-458. doi: 10.4172/1948-5948.1000324

57. El-Kadyl E, Moubasher MH (1982) Toxigenicity and toxin of Stachybotrys chartarum isolates from wheat straw samples in Egypt. J Exp Myco 6: 25-31.

58. Katz Y, Verleger H, Barr J, Rachmiel M, Kiviti S, et al. (1999) Indoor survey of moulds and prevalence of mould atopy in Israel. Clin Exp Allergy 29: 186-192.

59. NAS (1993) Pesticides in the diets of infants and children. National Academy Press, Washington, DC.

60. Centers for Disease Control and Prevention (CDC) (2015) Histoplasmosis

61. Wheat J, Wass J, Norton J (1984) Cavitary histoplasmosis occurring during two large urban outbreaks: analysis of clinical, epidemiologic, roentgenographic and laboratory features. Medicine (Baltimore) 63: 201-9.

62. Chapman SW, Lin AC, Hendricks A, Nolan RL, Currier MM, et al. (1997) Endemic blastomycosis in Mississippi: Epidemiological and clinical studies. Semin Respir Infect 12: 219-28.

63. Stevens DA (1995) Coccidioidomycosis. N Engl J Med 332: 1077-82.

64. Kowalski WJ (2000) Indoor mold health hazards and remediation. HPAC Engineering.

65. New York City Department (NYCD) of Health and Mental Hygiene (2008) Guidelines on assessment and remediation of fungi in indoor environment.

66. Adhikari A, Sen MM, Gupta-Bhattacharya S, Chanda S (2004) Airborne viable, non-viable and allergenic fungi in a rural agricultural area of India: A 2 year study at five outdoor sampling stations. Sci Total Environ 326: 123-141.

67. Hawksworth DL (1991) The fungal dimension of biodiversity: Magnitude, significance and conservation. Mycol Res 95: 641-655.

68. Albaum S, Masaphy S (2009) Comparison of rose bengal-chloramphenicol and modified aureomycin-rose bengal-glucose-peptone agar as media for the enumeration of molds and yeasts in water by membrane filtration techniques. $J$ Microbiol Methods 76: 310-312.

69. Carlile MJ, Watkinson SC, Gooday GW (2001) The fungi. Academic Press, London, England.

70. Amann R, Ludwig I, W, Schleifer KH (1995) Phylogenetic identification and in situ detection of individual microbial cells without cultivation. Microbiol Rev 59: 143-169.

71. MacNeil L, Kauri T, Robertson W (1995) Molecular techniques and their potential application in monitoring the microbiological quality of indoor air. Can J Microbiol 41: 657-665.

72. Dean TR, Kohan M, Betancourt D, Menetrez MY (2005).A simple polymerase chain reaction/restriction fragment length polymorphism assay capable of identifying medically relevant filamentous fungi. Mol Biotechnol 31: 21-28.

73. Gachon C, Saindrenan P (2004) Real-time PCR monitoring of fungal development in Arabidopsis thaliana infected by Alternaria brassicicola and Botrytis cinerea. Plant Physiol. Biochem 42: 367-371.

74. Haugland RA, Varma ML, Wymer J, Vesper SJ (2004) Quantitative PCR analysis of selected Aspergillus, Penicillium and Paecilomyces species. Syst Appl Microbiol 27: 198-210.

75. Vesper SJ, Wymer LJ, Meklin T, Varma M, Stott R, et al. (2005) Comparison of populations of mould species in homes in the UK and USA using mould-specific quantitative PCR. Lett Appl Microbiol 41: 367-373.

76. Bougoure DS, Cairney JW (2005) Assemblages of ericoid mycorrhizal and other root-associated fungi from Epacris pulchella (Ericaceae) as determined by culturing and direct DNA extraction from roots. Environ Microbiol 7: 819-827.

77. Schabereiter-Gurtner C, Pinar G, Lubitz W, Rolleke S (2001) Analysis of fungal communities on historical church window glass by denaturing gradient gel electrophoresis and phylogenetic 18S rDNA sequence analysis. J Microbiol Methods 47: 345-354.

78. Smit E, Leeflang P, Glandorf B, van Elsas JD, Wernars K (1999) Analysis of fungal diversity in the wheat rhizosphere by sequencing of cloned PCR-amplified genes encoding $18 \mathrm{~S}$ rRNA and temperature gradient gel electrophoresis. Appl Environ Microbiol 65: 2614-2621.

79. Buchan A, Newell SY, Moreta JI, Moran MA (2002) Analysis of internal transcribed spacer (ITS) regions of rRNA genes in fungal communities in a southeastern U.S. salt marsh. Microb Ecol 43: 329-340.

80. Yamamoto N, Nazaroff WW (2014) Assessing the aerodynamic diameters of taxon-specific fungal bioaerosols by quantitative PCR and next-generation DNA sequencing. J Aerosol Sci 78: 1-10.
81. Kennedy N, Brodie E, Connolly J, Clipson N (2006) Seasonal influences on fungal community structure in unimproved and improved upland grassland soils. Can J Microbiol 52: 689-694.

82. Meklin T, Haugland RA, Reponen T, Varma M, Lummus Z, et al. (2004) Quantitative PCR analysis of house dust can reveal abnormal mold conditions. J Environ Monit 6: 615-620.

83. Yike I, Allan T, Sorenson WG (1999) Highly sensitive protein translation assay for trichothecene toxicity in airborne particulates: comparison with cytotoxicity assays. App Environ Microbiol 65: 88-94.

84. Johanning E, Gareis M, Nielsen K, Dietrich R, Märtlbauer E (2002) Airborne mycotoxins sampling and screening analysis. Proceedings of the 9th International Conference on Indoor Air Quality and Climate (Indoor Air), Monterey, CA.

85. Bloom E, Bal K, Nyman E, Must A, Larsson L (2007) Mass spectrometry-based strategy for direct detection and quantification of some mycotoxins produced by Stachybotrys spp. and Aspergillus spp. in indoor environments. App Environ Microbiol 73: 4211-4217.

86. Wichmann GO, Herbarth (2002) The mycotoxins citrinin, gliotoxin and patulin affect interferon-gamma rather than interleukin-4 production in human blood cells. Environ. Toxicol 17: 211-218.

87. Johannessen LN, Nilsen AM, Løvik M (2007) Mycotoxin-induced depletion of intracellular glutathione and altered cytokine production in the human alveolar epithelial cell line A549. Toxicol Lett 168: 103-112.

88. Górny RL, Reponen T, Willeke K, Schmechel D, Robine E, et al. (2002) Fungal fragments as indoor air biocontaminants. Appl Environ Microbiol 68: 3522 3531.

89. Kildesø J, Wurtz H, Nielsen KF, Kruse P, Wilkins K, et al. (2003) Determination of fungal spore release from wet building materials. Indoor Air 13: 148-155.

90. Sorenson WG, Frazer DG, Jarvis BB, Simpson J, Robinson VA (1987) Trichothecene mycotoxins in aerosolized conidia of Stachybotrys atra. Appl Environ Microbiol 53: 1370-1375.

91. Cho SH, Seo SC, Schmechel D, Grinshpun SA, Reponen T (2005) Aerodynamic characteristics and respiratory deposition of fungal fragments. Atmos Environ 39: 5454-5465.

92. Green B, Tovey E, Sercombe J, Blachere F, Beezhold D, et al. (2006) Airborne fungal fragments and allergenicity. Med Microbiol 44: S245-S255.

93. United States Environmental Protection Agency (EPA) (2008) Mold remediation in schools and commercial buildings.

94. Rao CY, Burge HA (1996) Review of quantitative standards and guidelines for fungi in indoor air. J Air Waste Manage 46: 899-908.

95. Prahl P (1992) Reduction of indoor airborne mould spores. Allergy 47: 362-365

96. Kercsmar C, Dearborn D (2006) Reduction in asthma morbidity in children as a result of home remediation aimed at moisture sources. Environ Health Perspect 114: 1574-1580.

97. Barkai-Golan R (1957) A study of air borne fungi in Israel. Int J Biometeorol 1 : 83-86.

98. Sun Y, Sundell J, Zhang Y (2007) Validity of building characteristics and dorm dampness obtained in a self-administrated questionnaire. Sci Total Environ 387: 276-282.

99. Cooley JD, Wong WC, Jumper CA, Straus DC (1998) Correlation between the prevalence of certain fungi and sick building syndrome. Occup Environ Med 55: 579-584.

100. Engman LH, Bornehag CG (2004) Dampness in buildings and health report from an on-going epidemiological investigation in Sweden. Indoor Air (Supplement 14) 7: 59-66.

101.Dales R, Miller D, White J, Dulberg C, Lazarovits Al (1998) Influence of residential fungal contamination on peripheral blood lymphocyte populations in children. Arch Environ Health 53: 190-195

102. Fukuhara F, Saito J, Sato S, Sato Y, Nikaido T, et al. (2011) A validation study of asthma screening criteria based on subjective symptoms and fractional exhaled nitric oxide. Annals of Allergy, Asthma and Immunology 107: 480-486.

103. Kolarik B, Lagercrantz L, Sundell J (2009) Nitric oxide in exhaled and aspirated nasal air as an objective measure of human response to indoor air pollution. Indoor Air 19: 145-152. 
Citation: Masaphy S, Ezra R (2016) Targeted Inspection of Environmental Mycological Load for Mitigation of Indoor Mold toward Improved Public Health. J Microb Biochem Technol 8: 449-458. doi: 10.4172/1948-5948.1000324

104. Amirav I, Zacharasiewicz A (2008) Non-invasive monitoring of inflammation in asthma using exhaled nitric oxide. Israeli Medical Association Journal 10: 146-148.

105. Vesper S, Wymer L (2016) The relationship between environmental relative moldiness index values and asthma. Int J Hygiene Environ Health 219: 233238.

106. Wanless D (2004) Securing good health for the whole population. London: HM Treasury 